\title{
AVALIANDO O CONTROLE ESTATÍSTICO DE QUALIDADE DO FLUXO AÉREO DO AEROPORTO SANTA MARIA ARACAJU-SE
}

\author{
Daiane Costa Guimarães \\ Universidade Federal de Sergipe (UFS) - Av. Marechal Rondon, s/n Jardim Rosa Elze - \\ CEP 49100-000 - São Cristóvão/SE \\ dayaned10@hotmail.com \\ Suzana Leitão Russo \\ Universidade Federal de Sergipe (UFS) - Av. Marechal Rondon, s/n Jardim Rosa Elze - \\ CEP 49100-000 - São Cristóvão/SE \\ suzana.ufs@hotmail.com
}

\begin{abstract}
Resumo
O gráfico de controle é um método de detecção das causas assinaláveis, ou seja, causas incomuns que, agindo no processo, provocam alta variabilidade na saída (resultado do processo). Este estudo tem como finalidade de avaliar o controle estatístico de qualidade por meio dos gráficos de controle de média - $\bar{X}$ e do desvio padrão - S. com o objetivo de verificar o fluxo aéreo do aeroporto de Aracaju-SE, as variáveis estudadas são: embarques, desembarques, pousos e decolagens dos anos de 2007 a 2012. As variáveis utilizadas para esse estudo foram cedidas pelo aeroporto junto à administração da INFRAERO. Foi feita a análise descritiva das variáveis estudadas. O software utilizado para as análises dos resultados foi o STATISTICA 11. Foram feito os gráficos de controle e nota-se que houve pontos fora de controle estatístico, assim verifica-se as autocorrelações dos dados, e depois aplica os modelos Box e Jenkins - ARIMA para que a serie se torne estacionária e assim retirar as autocorrelações dos dados. A técnica proposta, de primeiro retirar a autocorrelação dos dados para depois refazer os gráficos mostrou-se satisfatória estatisticamente. E com os resíduos obtidos nos modelos, foi feito os gráficos revisados, mostrando-se que todos os pontos estão dentro do limite de controle, demonstrando que o processo está sob controle estatístico de qualidade.
\end{abstract}

Palavras-chave: Aeroporto, Fluxo aéreo, Gráfico de Controle.

\begin{abstract}
The control chart is a method of detecting assignable causes, or unusual causes, acting in the process, lead to high variability in the output (process result). This study aims to evaluate the statistical quality control through the media control charts - $\mathrm{X}^{-}$and standard deviation - S. in order to verify the airflow of Aracaju-SE airport, the variables are: shipments, landings, takeoffs and landings for the years 2007 to 2012. The variables used for this study were provided by the airport by the administration of INFRAERO. It was made a descriptive analysis of the variables studied. The software used for analysis of the results was the STATISTICA 11. We made the control charts and notices that there were points out of statistical control, so there is the autocorrelations of the data, and then applies the Box and Jenkins models - ARIMA so the series becomes stationary and thus remove the autocorrelations of data. The technical proposal, first remove the autocorrelation of the data and then redo the graphics was statistically satisfactory. And with the waste obtained in models, it was made the revised charts, showing that all points are within the control limit, demonstrating that the process is in statistical quality control.
\end{abstract}

Keywords: Airport, air flow, control chart 


\section{INTRODUÇÃO}

Atualmente, a disponibilidade de aeroportos e a cobertura da malha aérea doméstica mostram-se, de maneira geral, adequadas, com distribuição que espelha a da população. As companhias aéreas nacionais mais representativas encontram-se financeiramente saudáveis e possuem relevantes planos de expansão. Nos últimos anos, o gradual processo de liberalização tarifária promovido pela ANAC (Agência Nacional de Aviação Civil) tornou o setor mais dinâmico e competitivo, e esse aumento de competitividade trouxe benefícios aos passageiros, que viram o preço médio por quilômetro voado baixar $48 \%$ entre 2003 e 2008 [1].

O Aeroporto de Santa Maria (Sergipe), está situado em uma área de mais de 5 milhões de metros quadrados. Diariamente, cerca de 6 mil pessoas circulam pelo terminal. Mais 900 funcionários trabalham no complexo aeroportuário para atender uma média mensal de $100 \mathrm{mil}$ passageiros. Possuem 48 vôos diários, 24 vôos chegam e 24 vôos partem. Nos meses de Junho e Julho, devido às comemorações das festividades juninas e período de férias, o aeroporto recebe um considerável aumento de vôos charter [2].

$\mathrm{O}$ aumento da demanda do transporte aéreo nos últimos anos tem obrigado aeroportos do mundo todo a realizar grandes investimentos para aumentar a capacidade, melhoria da eficiência e controle dos congestionamentos. Em resposta a esta tendência, tem-se utilizado ferramentas de suporte a tomada de decisões [3].

Com os dados do aeroporto de Aracaju - SE, usando as variáveis:embarque, desembarque, pouso e decolagem, aplicam-se os Gráficos de Controle de Shewhart para identificar os pontos aberrantes, sendo verificados os limites, os quais indicarão se os dados estão ou não "sob controle". Caso haja dado fora do limite, será considerada irregularidade, um possível outliers. Após serem detectados os pontos fora do limite de confiança tais valores serão removidos.

\section{REVISÃO TEÓRICA}

\subsection{Aeroporto de Santa Maria}

O aeroporto Santa Maria, em Aracaju, registrou o maior crescimento no fluxo de passageiros no primeiro semestre desse ano. Foi registrado um crescimento de 26,3\%, calculados de janeiro a maio de 2012, em relação ao mesmo período do ano de 2011. Segundo o superintendente da Infraero em Sergipe, Luiz Alberto Bittencourt, dois setores impulsionaram o crescimento no aeroporto de Aracaju. "Esse aumento se deve às ações de divulgação do destino em outros estados, como também o crescimento da economia sergipana. Esses dois setores impulsionaram a movimentação do Aeroporto Santa Maria no primeiro semestre de 2012" [4].

\subsection{Controle de Qualidade}

Os gráficos de controle estatístico de processos mais conhecidos e amplamente aplicados no setor industrial são ainda, sem dúvida, os tradicionais gráficos de Shewhart. No Brasil, por exemplo, o mais comum deles (o par de gráficos X e R), que mostra a média de vários subgrupos racionais de observações sucessivas, é enfatizado na maioria das literaturas de controle de qualidade existentes como se não houvesse nenhum outro tipo de gráfico [5].

Segundo Shewhart (1931) argumentou com a experiência que o uso de três limites sigma fazia sentido econômico. O desvio padrão tem de ser calculada a partir dos dados. É convencional não para estimar o desvio padrão, através da aplicação da fórmula habitual para o desvio padrão da amostra para o conjunto de 25 medições, mas sim através de um método baseado no "local", em vez de variabilidade "global". O raciocínio para esta aproximação é que, se os dados preliminares incluem qualquer variação causa especial que o criador não tem conhecimento da carta, em seguida, a utilização de tais métodos atenua o efeito prejudicial que tal variação especial causa pode ter sobre a localização dos limites do gráfico [6]. 


\subsection{Tipos de Gráficos de Controle}

O gráfico de controle é um método de detecção das causas assinaláveis, ou seja, causas incomuns que, agindo no processo, provocam alta variabilidade na saída (resultado do processo). Por meio do gráfico de controle é possível a avaliação de tendências, padrões de não aleatoriedade e instabilidades do processo, permitindo a sua interrupção e a ação corretiva antes que se produzam itens fora dos limites de especificação (MONTGOMERY, 1997)[7].

Os dois tipos mais comuns de gráfico de controle são os de variáveis e os de atributos. No primeiro caso, quando as características do processo podem ser expressas em uma medida numérica [8]. Mas para o segundo caso, temos que nem sempre é possível por meio de medidas numéricas expressarem o processo [9].

No trabalho abordado os gráficos $\bar{X}-S$, vão ser preferíveis em relação aos gráficos $\bar{X}-R$, quando $\mathrm{n}>10$ ou 12 já que para amostras maiores a amplitude amostral $\mathrm{R}$ perde eficiência para estimar $\sigma$, lembrando que um dos pressupostos para aplicação dos mesmos seria a normalidade dos dados [10].

\section{a) Gráficos $\bar{X}-S$}

Esses gráficos funcionam como os gráficos $\bar{X}$ e R, porém permitem trabalhar com subgrupos com mais de dez elementos. A diferença em relação aos gráficos $\bar{X}$ e $\mathrm{R}$ estão na forma como a variação de cada subgrupo é calculada. Nos gráficos $\bar{X}$ e R ela é obtida pela média dos valores de cada subgrupo. Nos gráficos $\bar{X}$ e $S$, por sua vez, ela é obtida pelo desvio padrão de cada subgrupo [11].

Expressões para o cálculo dos limites de controle dos gráficos $\bar{X}-S$ :

\section{* Gráfico $\bar{X}$ :}

$$
\begin{aligned}
\mathrm{LSC}= & \overline{\bar{x}}+\frac{3 \bar{s}}{c_{4} \sqrt{n}}=\overline{\bar{x}}+A_{3^{\bar{s}}} \\
& \mathrm{LM}=\overline{\bar{x}}, \quad(2) \\
\mathrm{LIC}= & \overline{\bar{x}}-\frac{3 \bar{s}}{c_{4} \sqrt{n}}=\overline{\bar{x}}-A_{3^{\bar{s}}}
\end{aligned}
$$

Onde $A 3=3 / c_{4} \sqrt{n}$ é uma constante tabelada em função do tamanho $n$ das amostras.

* Gráfico $S$ :

$$
\begin{gathered}
\mathrm{LSC}=\bar{s}+3 \hat{\sigma}_{s}=B_{4} \bar{s}, \\
\mathrm{LM}=\bar{s}, \\
\mathrm{LIC}=\bar{s}-3 \hat{\sigma}_{s}=B_{3} \bar{s}
\end{gathered}
$$

Onde $\hat{\sigma}_{s}$ é a estimativa do desvio padrão da distribuição de $s$ e $B 3$ e $B 4$ são constantes tabeladas em função do tamanho $n$ das amostras [10].

Resumindo temos que,

* Para as médias:

Limite Superior de Controle:

$$
\begin{gathered}
\text { LSC }=\overline{\bar{X}}+A_{2} * \bar{R} \\
\text { Linha Central: } \\
\text { LC }=\overline{\bar{X}}
\end{gathered}
$$

Limite Inferior de Controle:

$$
\mathrm{LIC}=\overline{\bar{X}}-A_{2} * \bar{R}
$$

* Para as amplitudes:

$$
\begin{aligned}
& \text { Limite Superior de controle: } \\
& \text { LSC }=D_{4} * \bar{R} \\
& \text { Linha Central: } \\
& \text { LC }=\bar{R} \\
& \text { Limite Inferior de Controle: } \\
& \text { LIC }=D_{3} * \bar{R}
\end{aligned}
$$

As fórmulas para o cálculo dos limites dos tipos de gráficos de controle para variáveis. Onde os valores A2, A3, B3, B4, D3 e D4 são tabelados e dependentes dos números de elementos no subgrupo racional. 


\section{METODOLOGIA}

Primeiramente foi feita a coleta dos dados mensais de pouso, decolagem, embarques e desembarques dos anos de 2007 a 2012 na administração do aeroporto de Aracaju junto com a INFRAERO. O conjunto de dados analisados trata-se de informações do fluxo aéreo. $\mathrm{O}$ procedimento utilizado nesse estudo fazer parte de uma revisão da literatura do controle estatístico de qualidade, onde serão levantadas as informações teóricas a respeito dos gráficos de controle. O software utilizado para as análises e gráficos foi o STATISTICA 11.

\section{RESULTADOS E DISCUSSÕES}

\subsection{Aplicação dos Gráficos de Controle de Shewhart para a variável embarque no período de 2007 A 2012}

\subsubsection{Aplicando os gráficos de controle para a variável embarque}

Montgomery (2000) comenta que a principal desvantagem de qualquer Gráfico de Controle de Shewhart é usar somente informação sobre o processo contido do último ponto demarcado e ignorar qualquer informação dada pela sequência inteira de pontos. Essa característica faz com que o gráfico de controle de Shewhart seja insensível a pequenos e contínuos desvios no processo, da ordem de até 1,5 desvios padrão.

Pode-se constatar no gráfico de controle de Shewhart da média e desvio padrão, figura 1, que existem alguns dos pontos representados situa-se fora dos limites de controle, sendo alguns acima do limite superior e também abaixo do limite inferior. A explicação desses pontos fora do controle estatístico de qualidade deve ta relacionada com os acidentes aéreos, crise mundial, logo após esses acontecimentos as companhias aéreas baixou o preço das passagens assim ocorreu um aumento no número de passageiros.

Existe uma forte presença autocorrelação nos dados da média, assim a serie é estacionária em variância e não é estacionária em média.

Figura 1- Gráfico de Shewhart para média e desvio padrão

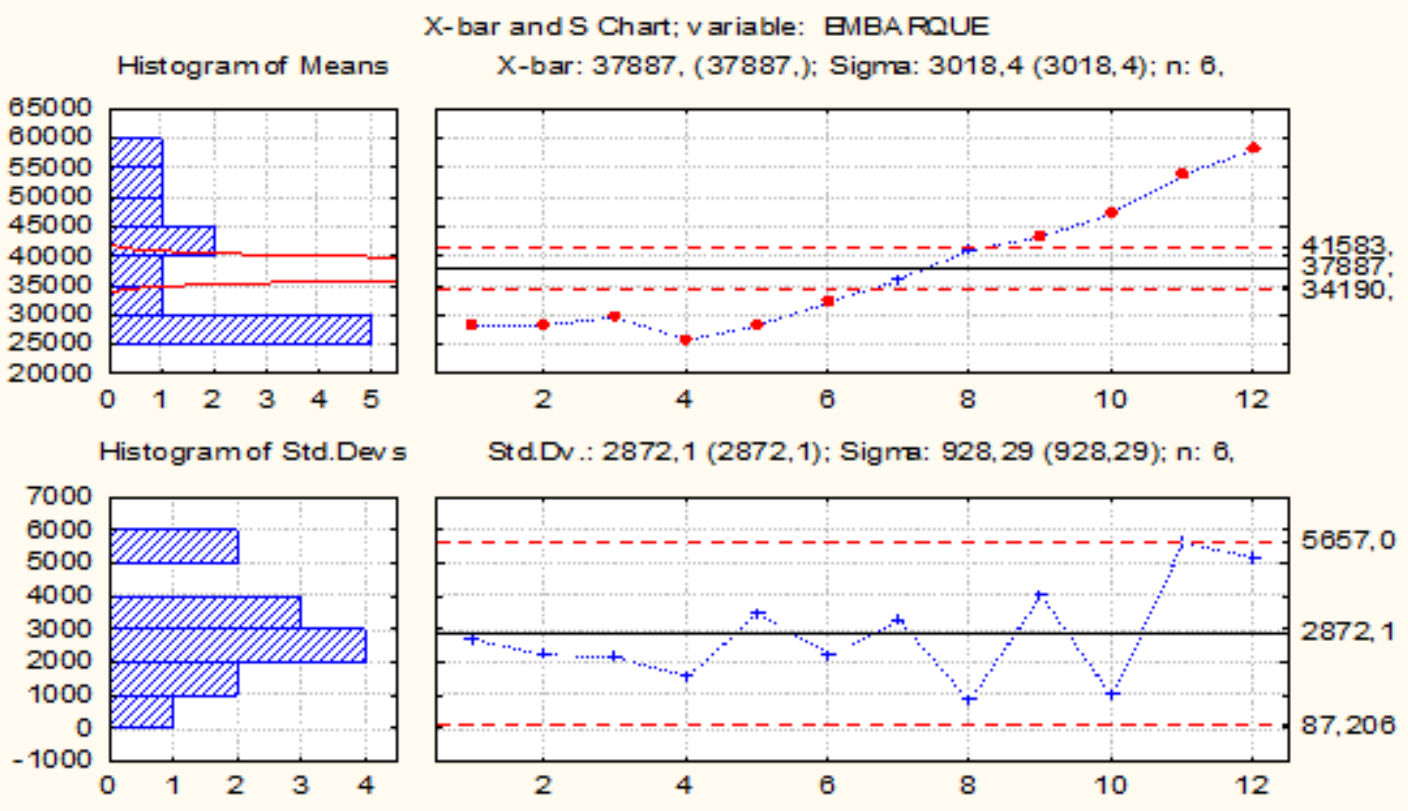

Fonte: Autoria Própria 


\subsubsection{Aplicando os gráficos de controle para a variável embarque revisados}

Na figura 2, após ter feito uma modelagem ARIMA com intervenção nos dados da variável embarque, foi retirados as análises dos resíduos para ser feito o gráfico de controle Shewhart revisado.

Observa-se que todos os pontos estão dentro do limite de controle, fazendo assim, que os dados estão sobre controle estatístico de qualidade, mostrando que a ocorre uma estacionariedade tanto na média quanto no desvio padrão.

\section{Figura 2 - Gráfico de Shewhart para média e desvio padrão revisado da variável embarque}

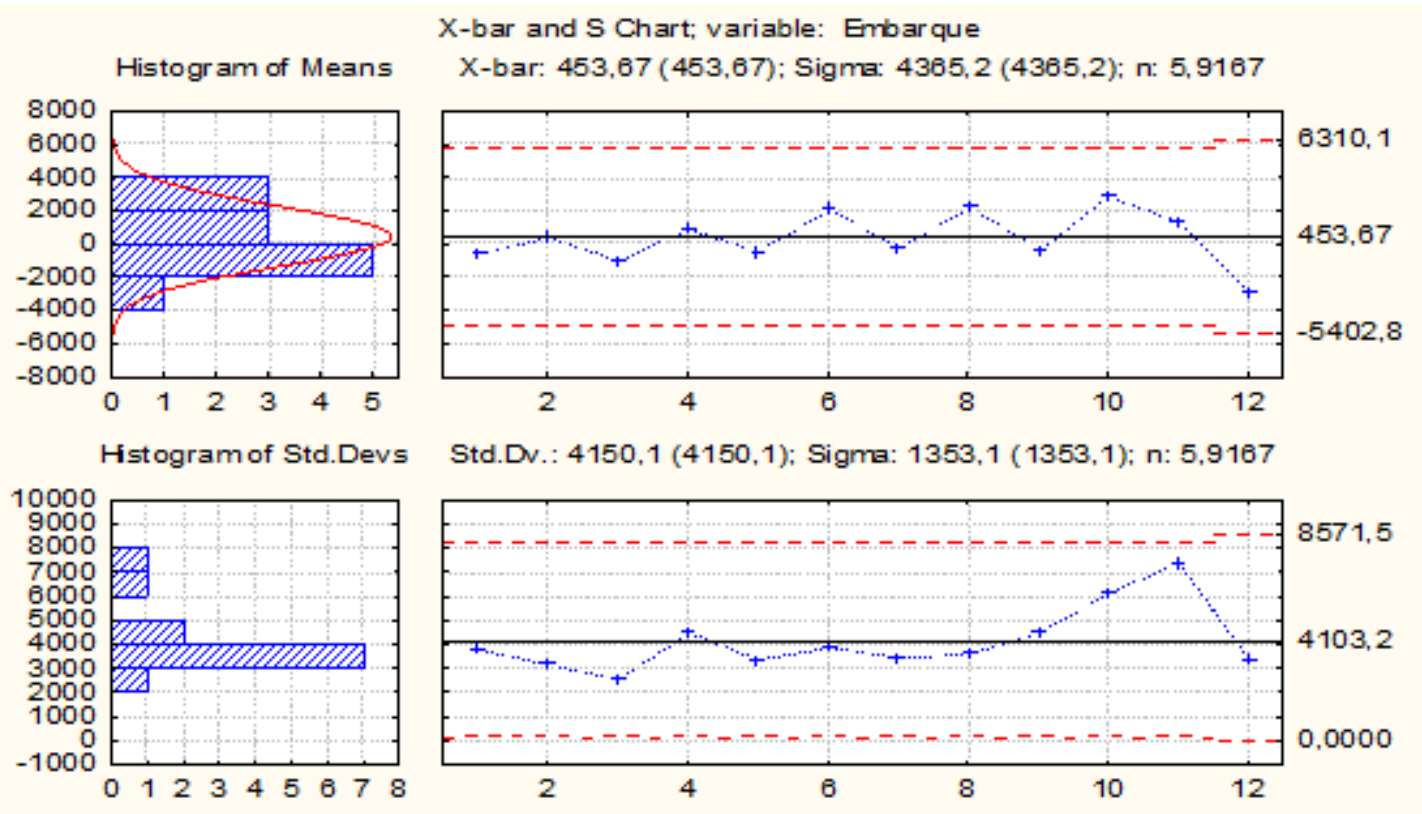

Fonte: Autoria Própria

\subsection{Aplicação dos gráficos de controle de Shewhart para a variável desembarque no período de 2007 a 2012}

\subsubsection{Aplicando os gráficos de controle para a variável desembarque}

Através da análise dos gráficos de controle, é possível ver que o processo não está sob controle estatístico, já que ocorreram alguns pontos fora de controle.

Considerados possíveis outliers os pontos que ultrapassam os limites superiores e inferiores (destacados em vermelho) da variável estudada, e explicação disso pode ter provocado por casa dos acidentes aéreos, crise mundial etc. Logo após esses acontecimentos as companhias aéreas baixaram o preço das passagens assim ocorreu um aumento no número de passageiros. Assim, precisa ser feito o gráfico revisado (Figura 3). 
Figura 3- Gráfico de Shewhart para média e desvio padrão da variável desembarque

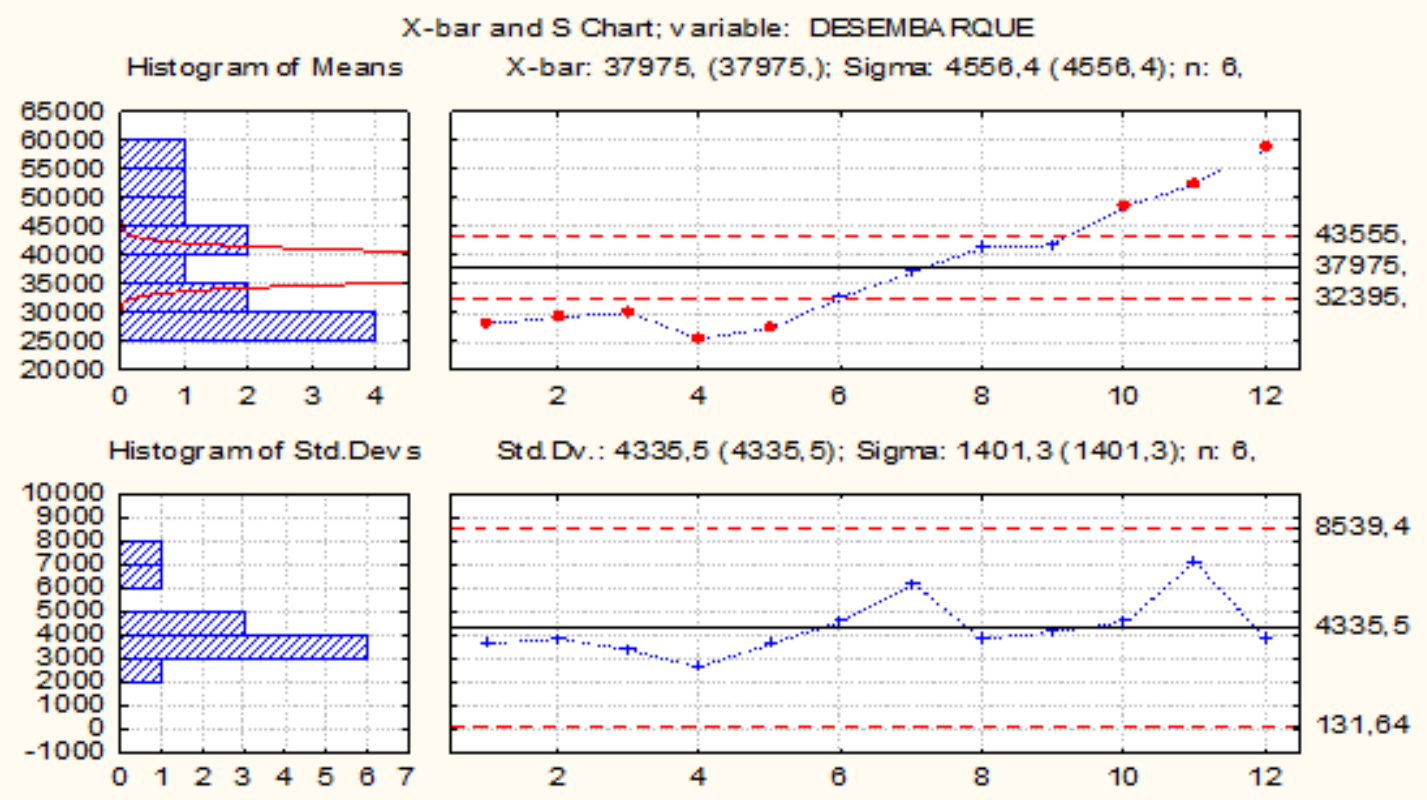

Fonte: Autoria Própria

\subsubsection{Aplicando os gráficos de controle para a variável desembarque revisados}

Primeiramente foi feita uma modelagem ARIMA com intervenção na série para que a tal se torne estacionária e assim ser feito os gráficos de controle. Foi feito vários modelagens ARIMA com intervenção, quando encontrado um modelo adequado pra a serie foi retirada as análises dos resíduos e por fim ser feito os gráficos de controle revisado (Figura 4).

Pode-se notar nos gráficos acima que os dados são estacionários em média e estacionários em desvio padrão, pois todos os pontos apresentam dentro do controle estatístico de qualidade.

\section{Figura 4 - Gráfico de Shewhart para média e desvio padrão revisado da variável desembarque}

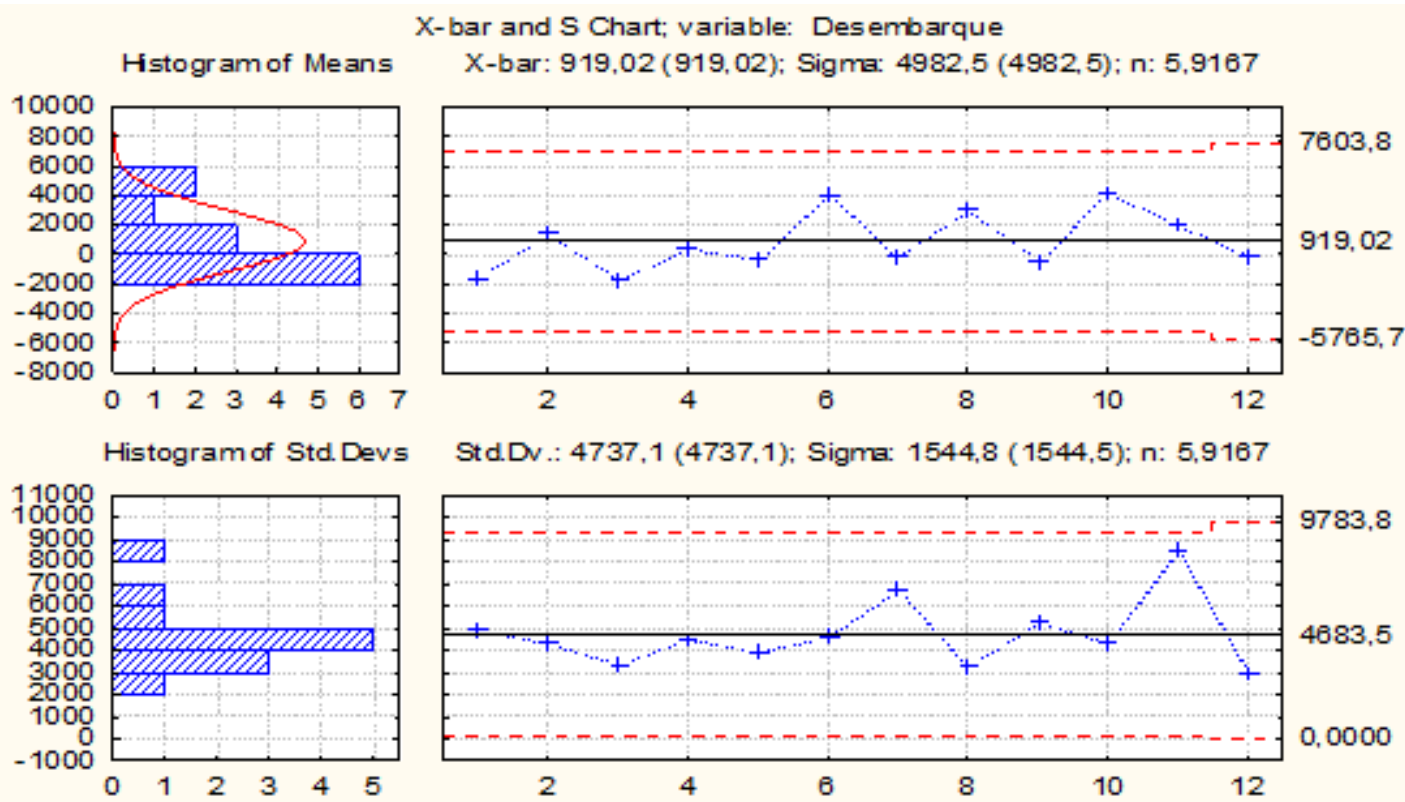

Fonte: Autoria Própria 
4.3 Aplicação dos gráficos de controle de Shewhart para a variável pouso no período de 2007 a 2012

\subsubsection{Aplicando os gráficos de controle para a variável pouso}

Pode-se constatar no Gráfico de Controle de Shewhart, figura 5, que 7 dos pontos representados situam-se fora dos limites de controle, sendo 3 pontos acima do limite superior e 4 pontos abaixo do limite inferior.

Estes pontos que apresentam fora do limite de controle são causados por alguns motivos, são eles: Devido os acidentes aéreos ocorrido no Brasil nos meados de 2007 ouve uma queda de aeronaves, pois muitos passageiros ficaram com medo de viajar, assim, as agências aéreas diminuíram o preço das passagens fazendo com que as pessoas voltassem a viajar, então logo após esses acontecimentos teve um aumento de pouso.

\section{Figura 5 - Gráfico de Shewhart para média e desvio padrão da variável pouso}

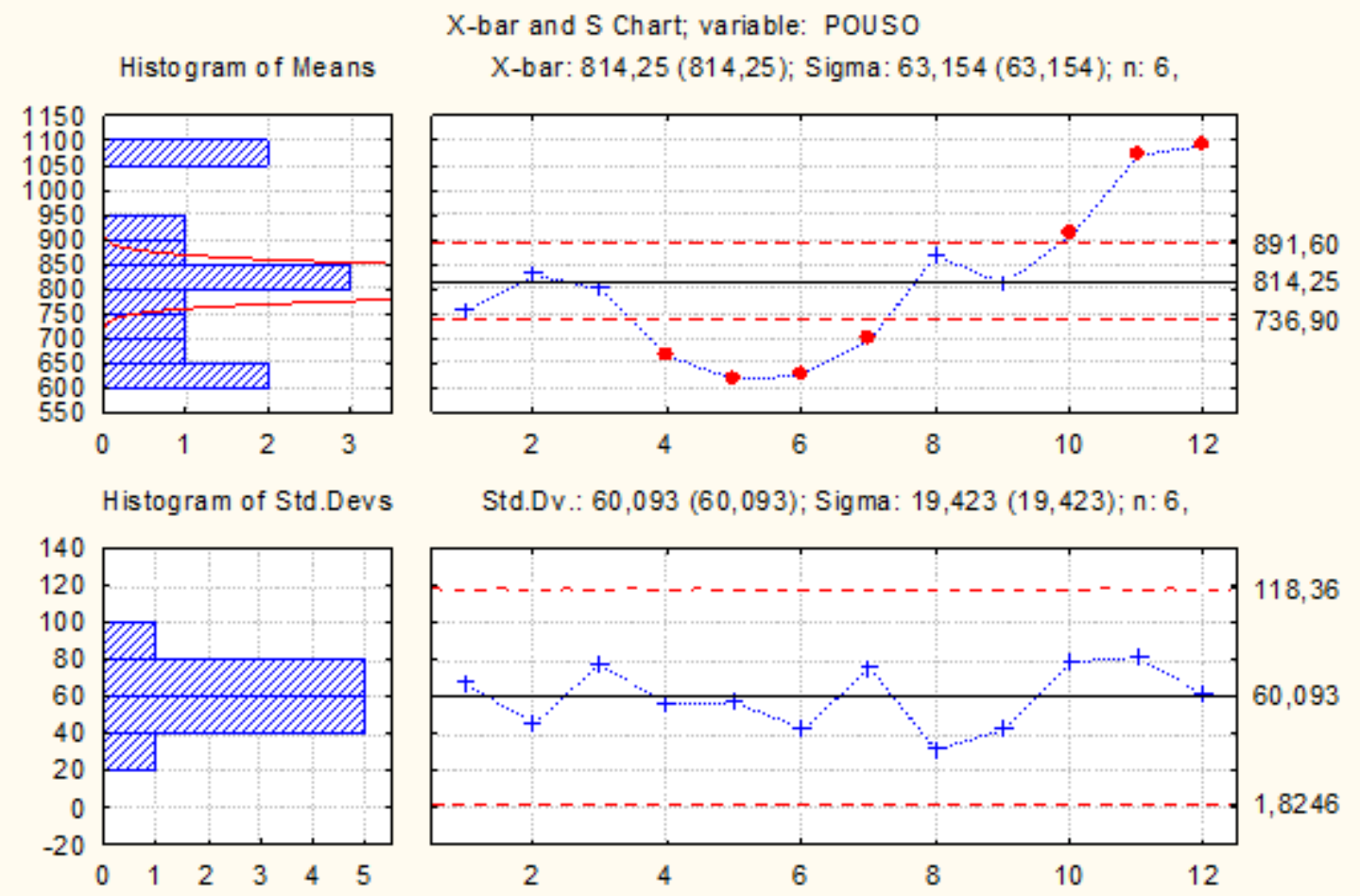

Fonte: Autoria Própria

\subsubsection{Aplicando os gráficos de controle para a variável pouso revisados}

Inicialmente foi feita vários modelagens ARIMA com intervenção nos dados da variável pouso para que a tal se torne estacionária e assim ser feito os gráficos de controle. Quando encontrado um modelo adequado pra a serie foi retirada as análises dos resíduos e por fim ser feito os gráficos de controle revisado. (Figura 6). O gráfico da média e desvio padrão está sobre controle estatístico de qualidade. 
Figura 6 - Gráfico de Shewhart para média e desvio padrão revisado da variável pouso

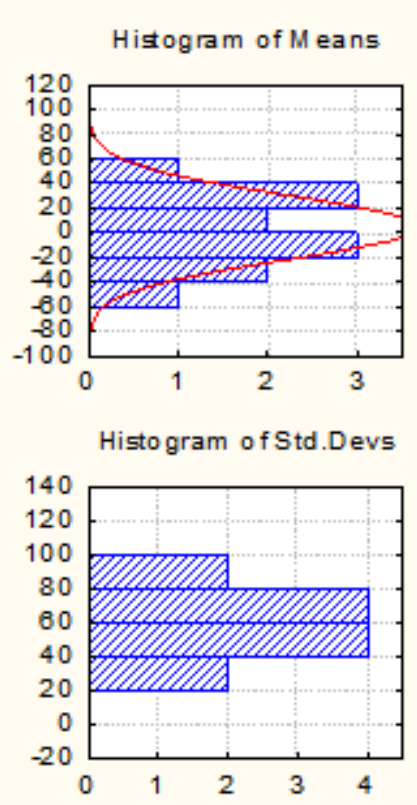

$\mathrm{X}$-bar and S Chart; variable: Pouso

X-bar. $4,4330(4,4330)$, Sigma: $63,575(83,575)$, n: 5,9167

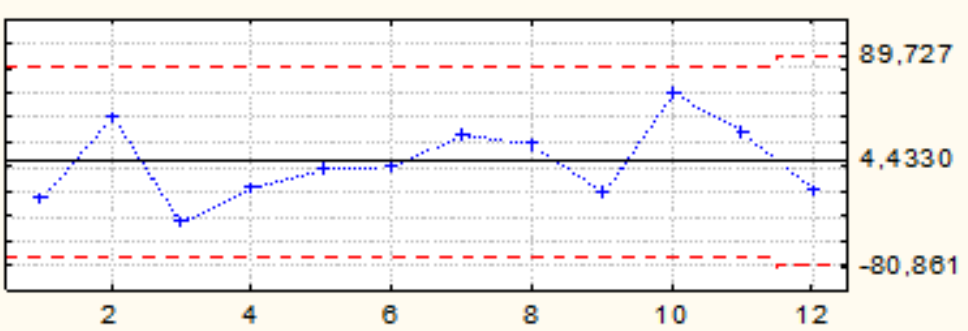

Std. Dv.: $60,443(60,443)$; Sigma: $19,702(19,707) ; n: 5,9167$

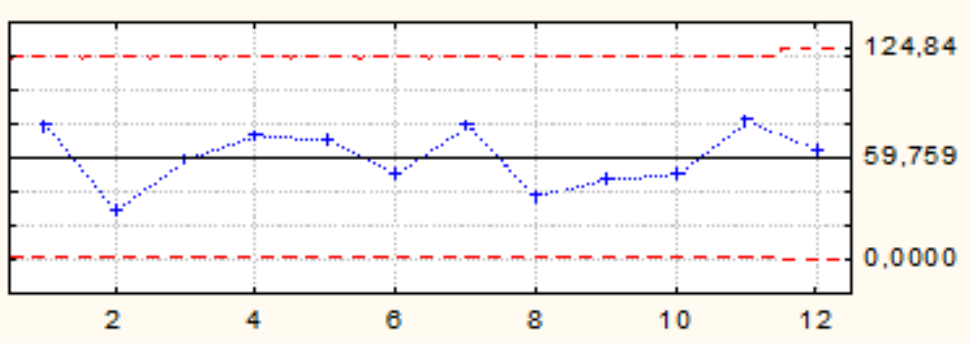

Fonte: Autoria Própria

\subsection{Aplicação dos gráficos de controle de Shewhart para a variável decolagem no período de 2007 a 2012}

\subsubsection{Aplicando os gráficos de controle para a variável decolagem}

Foram realizados os gráficos de controle. Observa-se na figura 7 serão considerados possíveis outliers os pontos que ultrapassam os limites superiores e inferiores (destacados em vermelho). Verifica-se também que as maiorias das amostras se concentram abaixo do limite central, limite inferior. A explicação pode ter sido causada, devido os acidentes aéreos ocorrido no Brasil nos meados de 2007 ouvem uma queda de aeronaves, pois muitos passageiros ficaram com medo de viajar, assim, as agência aéreas diminuíram o preço das passagens fazendo com que as pessoas voltassem a viajar, então logo após esses acontecimentos teve um aumento de pouso. 
Figura 7 - Gráfico de Shewhart para média e desvio padrão da variável decolagem

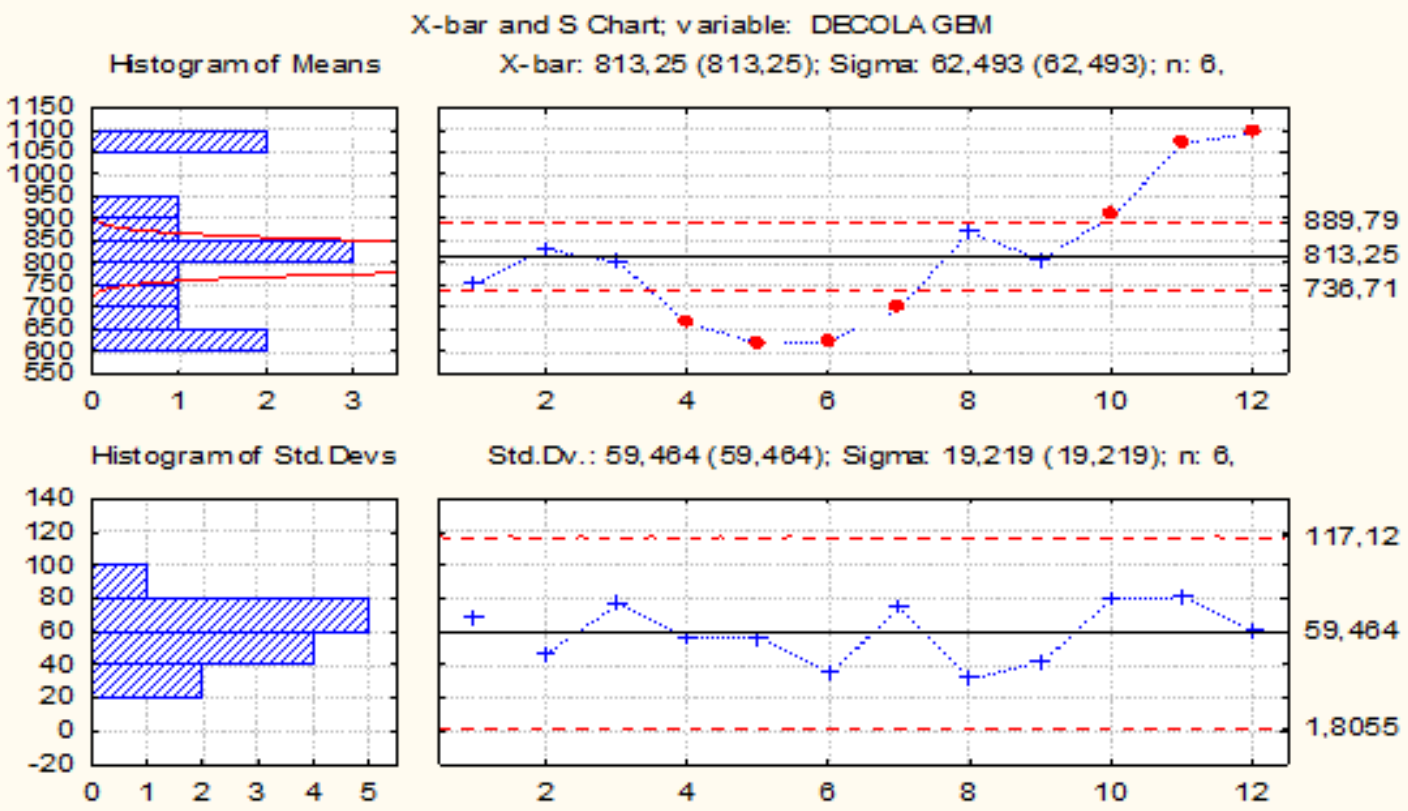

Fonte: Autoria Própria

\subsubsection{Aplicando os gráficos de controle para a variável decolagem revisados}

Depois de ter feito vários modelos ARIMA com intervenção, quando encontrado um modelo adequado pra a serie foi feito os gráficos revisados com os dados dos resíduos. $\mathrm{O}$ gráfico da serie revisada (figura 8), nota-se que o gráfico da média e desvio padrão, todos os pontos estão dentro do limite de confiança, portanto estacionário em média e desvio padrão.

Figura 8-Gráfico de Shewhart para média e desvio padrão revisado da variável decolagem

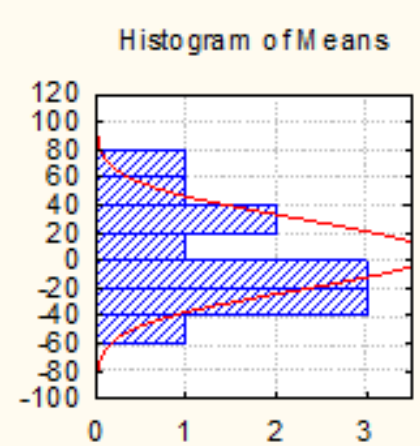

$\mathrm{X}$-bar and S Chart; variable: Decolagem

X-bar. $4,4018(4,4018)$; Sigma: $63,024(63,024)$; n: 5,9167

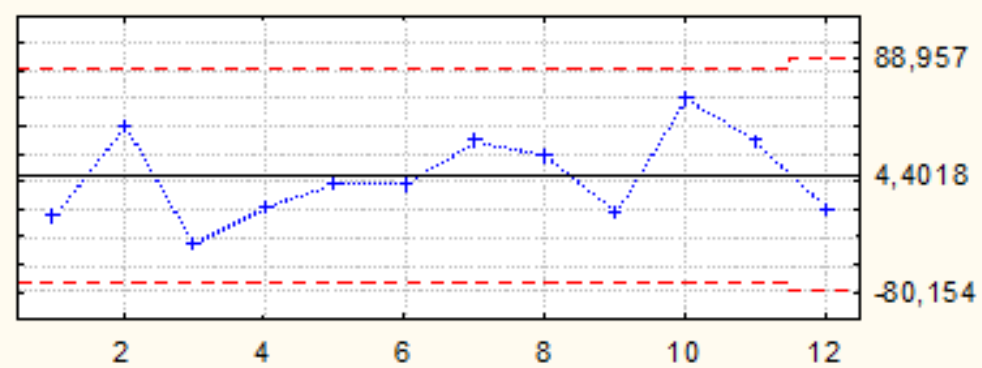

Histogram of Std.Devs

Std. Dv.: 59,920 (59,920); Sigma: $19,531(19,536)$; n: 5,9167
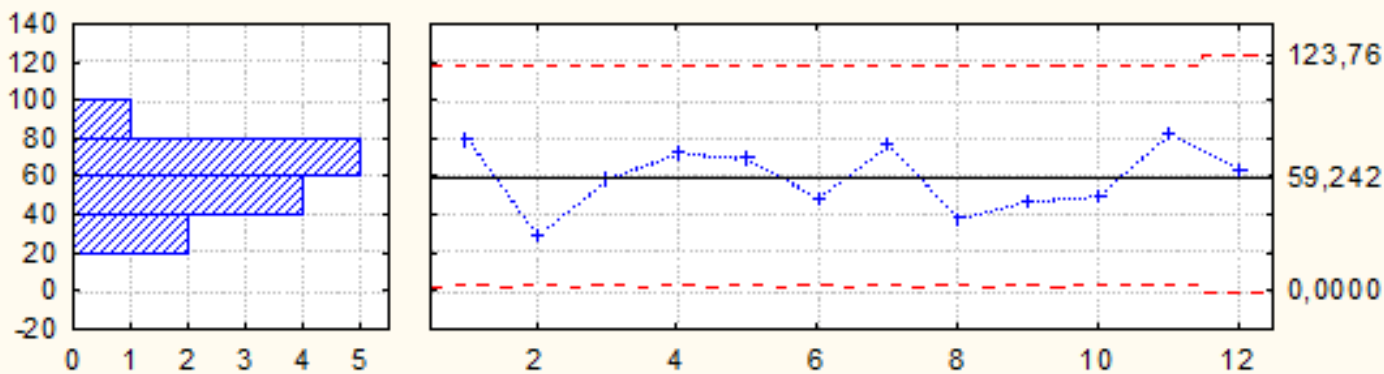

Fonte: Autoria Própria 


\section{CONCLUSÃO}

Segundo Montgomery (2004), vários critérios podem ser aplicados simultaneamente a um gráfico de controle, para se determinar se o processo está sob controle. O critério básico é um ou mais pontos fora dos limites de controle. Os critérios suplementares são, às vezes, usados para aumentar a sensitividade dos gráficos de controle há uma pequena mudança no processo, de modo a responder mais rapidamente a uma causa atribuível.

Este estudo buscou apresentar as técnicas tradicionais de Controle Estatístico de Qualidade, através dos gráficos de controle por variáveis no fluxo de aeronaves do aeroporto de Aracaju - SE. Para tal, foram coletados os dados mensais na administração do aeroporto, no período de 2007 a 2013.

Primeiramente foram verificadas as análises descritivas das variáveis: embarque, desembarque, pouso e decolagens. Percebendo-se que nos anos de 2008 e 2009 ocorreu uma queda dos números de aeronaves.

Foram feito os gráficos de controle para as variáveis abordadas, e observa-se que nos gráficos do desvio padrão houve uma estacionariedade da serie, já para a média houve pontos fora do controle estatísticos em ambos as variáveis, tais acontecimentos podem ter ocorrido por vários motivos, como por exemplo: o período em que o país viveu em situação de crise no sistema aéreo, o número de pouso e decolagem diminuiu consideravelmente, voltando a se estabilizar quando este foi resolvido. Verificou-se a autocorrelação dos dados, e assim foi retirada através dos modelos Box e Jenkins, modelos ARIMA.

Com os resíduos obtidos nos modelos foi refeito os gráficos, deste modo todos os pontos ficaram dentro do limite de controle para todas as variáveis estudadas.

\section{REFERÊNCIA}

[1] MCKINSEY \& COMPANY. Estudo do Setor de Transporte Aéreo do Brasil: Rio de Janeiro, 2010.

[2] Novos voos chegam em janeiro a Sergipe. Aeroporto Santa Maria vai receber a partir do mês de janeiro mais dois novos voos. (em português), Acessado: Emsergipe.com (24 de dezembro de 2011).

[3] CONFESSORE, G.; LIOTTA, G; GRIECO, R.A Simulation-Based Architecture for Supporting Strategic and Tactical Decisions in the Apron of Rome-Fiumicino Airport. Institute of Industrial Technologies and Automation, National Research Council of Italy, ITIA- CNR. Rome, Italy.2005 .

[4] INFRAERO. Aeroporto de Aracaju - Santa Maria. Disponível em: http://www.infraero.gov.br/index.php/br/aeroportos/sergipe/aeroporto-de-aracaju-santamaria.html. Acesso em: 10 ago. 2012

[5] ALVES, C.C. Gráficos de controle CUSUM: um enfoque dinâmico para a análise estatística de processos. Dissertação de Mestrado em Engenharia de Produção, UFSC, Florianópolis, 2003.

[6] SHEWHART, W.A. (1931) Economic Control of Quality of Manufactured Product. New York: D. Van Nostrand. Also available in a 50th anniversary edition published in 1980 by the American Society for Quality, Milwaukee, WI.

[7] MONTGOMERY, D. C. Introduction to Statistical Quality Control. 3rd ed. New York: JW, 1997.

[8] MONTGOMERY, D. C.. Introdução ao Controle Estatístico de Qualidade. LTC. 2009. [9] COSTA, A. F. B.,EPPRECHT, E. K. CARPINETTI, L. C. R..Controle Estatístico de Qualidade-2.ed. -4. Reimpr.- São Paulo: Atlas, 2010.

[10] RUSSO, S. L.; PRATA, M. S.; SIMON, V. H.. Aplicação do controle estatístico de qualidade na variável DT do processo de perfilagem de um poço de petróleo. Ingepro, 2010. 
[11] ROCHA, A. R. C., SOUZA, G. S., BARCELlOS, M. P. (2012), "Medição de Software e Controle Estatístico de Processos", PBQP Software, Brasília.

[12] MONTGOMERY, D. c. introdução ao controle estatístico da qualidade/ Douglas C. Montgomery; tradução Ana Maria Lima de Farias, Vera Regina Lima de Farias e Flores; revisão técnica Luiz da Costa Laurencel. 4. ed.reimpr. - Rio de Janeiro: LTC, 2004. 